\title{
Impacto del COVID-19 (SARS-CoV-2) a Nivel Mundial, Implicancias y Medidas Preventivas en la Práctica Dental y sus Consecuencias Psicológicas en los Pacientes
}

\author{
Impact of COVID-19 (SARS-CoV-2) Worldwide, Implications and Preventive \\ Measures in Dental Practice and its Psychological Consequences on Patients
}

Marcial Guiñez Coelho

GUIÑEZ, C. M. Impacto del COVID-19 (SARS-CoV-2) a nivel mundial, implicancias y medidas preventivas en la práctica dental y sus consecuencias psicológicas en los pacientes. Int. J. Odontostomat., 14(3):271-278, 2020.

RESUMEN: En diciembre de 2019, en China surgió un nuevo brote de neumonía la cual fue identificada como SARS-CoV-2 el cual tiene una rápida propagación al ser trasmitido principalmente mediante tos, estornudo o contacto. Presenta diversos síntomas como fiebre, tos seca y mialgia. Puede afectar a cualquier edad siendo principalmente leve y con resolución espontánea, pero en personas mayores y/o con comorbilidades puede presentar un curso severo o mortal. Para su diagnóstico es importante evaluar la presencia de fiebre, realizar una historia epidemiológica, TC de tórax y pruebas de laboratorio como RT-PCR o muestras del tracto respiratorio.Se realizó una revisión sistemática cualitativa realizando una búsqueda en las bases de datos Medline y ClinicalKey, se encontraron 72 artículos, los cuales se leyeron por completo, de ellos 16 cumplieron los criterios de inclusión y exclusión. La gran parte de las acciones odontológicas producen gotas o aerosoles, por esta razón se deben tomar medidas de protección, selección de pacientes y tipos de tratamientos para disminuir la posibilidad de infecciones en el personal de salud y pacientes, evaluar el uso de goma dique y colutorios previos a la atención, evitar instrumental generador de aerosoles, postergar atenciones electivas, tratar solamente emergencias dentales y extremar las medidas de desinfección de las instalaciones utilizando agentes biocidas efectivos contra el coronavirus. La trasmisión por fómites y aerosoles está comprobada por lo que debemos extremar precauciones. En situaciones de pandemia hay que considerar entregar un soporte psicológico a los pacientes, especialmente con patologías orales psicosomáticas las que pueden empeorar. La posibilidad de trasmisión en el periodo de recuperación no está comprobada ni descartada.

PALABRAS CLAVE: COVID-19, SARS-CoV-2, Coronavirus, Dentistry, Public Health.

\section{INTRODUCCIÓN}

En diciembre del 2019, en la ciudad de Wuhan, China, surgió un nuevo brote de neumonía de origen desconocido el cual se ha extendido rápidamente por todo el mundo (Ge, et al., 2020; Guo et al., 2020; Kampf et al., 2020; Li et al., 2020; Meng et al., 2020; Pan et al., 2020; Peng et al., 2020; Qu \& Zhou, 2020; Sohrabi et al., 2020; Tang et al., 2020; Van Doremalen et al., 2020; Xu et al., 2020). Según el reporte de la Organización Mundial de la Salud (OMS) al 25 de marzo hay un total de 413.467 casos confirmados y 18.433 muertes en el mundo, afectando a 197 países, siendo descrito como una pandemia y el mayor desafío de salud pública en el mundo (Guo et al.; Meng et al.; Pan et al.; Sabino-Silva et al., 2020; World Health Organization, 2020).
Esta nueva neumonía logró ser aislada y su genoma fue secuenciado recibiendo el nombre de SARS-CoV-2 o COVID-19 (Ge et al.; Guo et al.; Huh et al., 2020; Kampf et al.; Meng et al.; Pan et al.; Peng et al.; Qu \& Zhou; Sabino-Silva et al.; Sohrabi et al.; Tang et al.; Van Doremalen et al.; Xu et al.), presentando una similitud genética de un $79 \%$ con el SARSCoV y un $50 \%$ con MERS-CoV, todos de la familia de los $\beta$-coronavirus (Ge et al.; Kampf et al.; Meng et al.; Peng et al.; Sabino-Silva et al.; Van Doremalen et al.). La mayoría de los coronavirus causan enfermedades respiratorias leves en humanos, sin embargo, los $\beta$ coronavirus causan enfermedades respiratorias fatales (Peng et al.). 
Este nuevo virus se caracteriza por una rápida propagación, superando a MERS-CoV y SARS-CoV, existe trasmisión directa persona a persona por inhalación de gotitas producidas al hablar en voz alta o reír ya que la saliva contiene virus en pacientes infectados; aunque falta corroborar la posible transmisión vertical entre madre y recién nacido (Ge et al.; Guo et al.; Kampf et al.; Li et al.; Meng et al.; Pan et al.; Peng et al.; Qu \& Zhou; Sabino-Silva et al.; Sohrabi et al.; Tang et al.; Van Doremalen et al.; Xu et al.).

\section{Vías de Trasmisión COVID-19 (Fig. 1).}

Los síntomas típicos son fiebre, tos seca, mialgia, fatiga y disnea con una tomografía torácica (TC) anormal observando neumonía bilateral, opacidad como vidrio esmerilado y/o sombras irregulares bilaterales. Los síntomas atípicos son producción de esputo, cefaleas, hemoptisis, diarrea, mareo, dolor abdominal, náuseas, vomito, confusión y dolor de garganta (Ge et al.; Meng et al.; Peng et al.; Sabino-Silva et al.; Sohrabi et al.; Xu et al.).

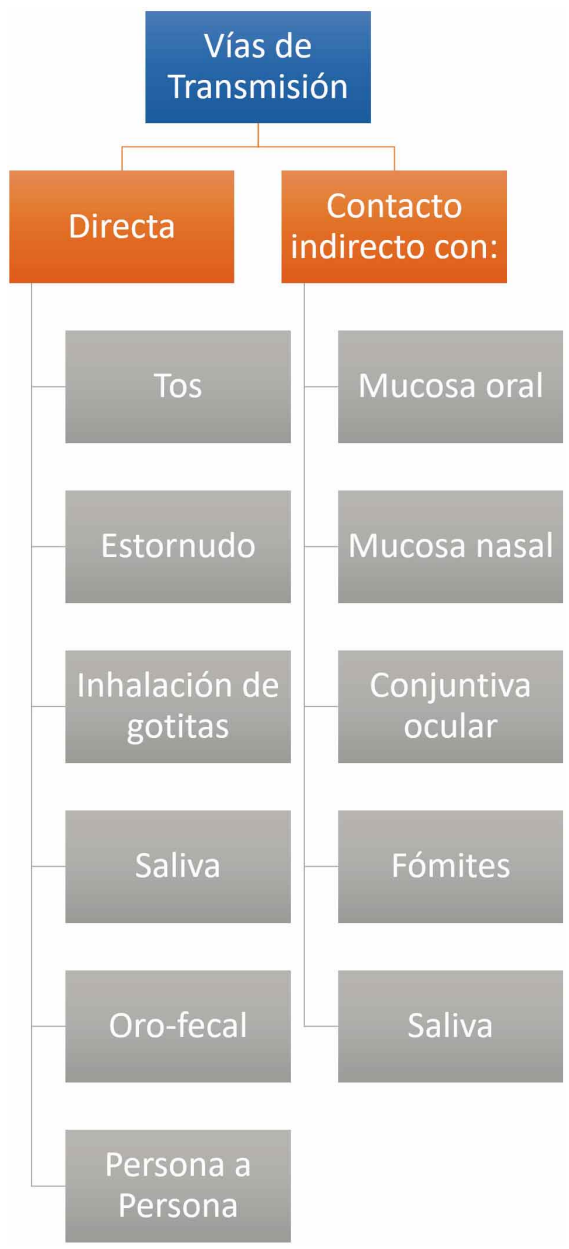

Fig. 1. Vías de transmisión del COVID-19.
Los datos epidemiológicos indican que todas las edades son susceptibles, pero presentan una mayor prevalencia los hombres con edad media de 56 años, individuos con contacto cercano con personas infectadas sintomáticas o asintomáticas, incluyendo otros pacientes que se encuentren en los centros de salud, al igual que el equipo médico quienes conforman un porcentaje importante de las personas infectadas. Las razones específicas de las fallas del equipo de protección personal (EPP) en el equipo de salud deben ser investigadas (Kampf et al.; Li et al.; Meng et al.; Peng et al.; Sabino-Silva et al.).

La mayoría de los casos son leves y se resuelven espontáneamente, según los datos en China, un $15 \%-25 \%$ de sus casos son severos (Meng et al.). Los pacientes de edad avanzada y/o con comorbilidad como diabetes mellitus (DM), hipertensión arterial (HTA), enfermedades cerebrovasculares, cardiacas, endocrinas, digestivas, respiratorias e inmunosuprimidos son más susceptibles a desarrollar una infección severa los pueden presentar daño renal agudo, síndrome de distrés respiratorio agudo, falla orgánica, falla respiratoria progresiva e incluso morir (Meng et al.; Peng et al.; Sabino-Silva et al.; Sohrabi et al.; Xu et al.).

El periodo de incubación va de 2 a 19 días, con una media de 5.2 días. Individuos en incubación o asintomáticos pueden trasmitir el virus. Estudios sugieren que se debe demostrar si los pacientes en fase de recuperación son una fuente potencial de trasmisión del virus (Ge et al.; Kampf et al.; Li et al.; Meng et al.; Pan et al.; Peng et al.; SabinoSilva et al.; Sohrabi et al.; Van Doremalen et al.; Xu et al.).

La tasa de mortalidad del SARS-CoV-2 a nivel global es de un $0.39 \%-17.9 \%$. Un ajuste realizado en los datos de Wuhan muestran una tasa de mortalidad del 20\% (Baud et al., 2020; Center for EvidenceBased Medicine, 2020; Ge et al.; Meng et al.; Sohrabi et al.). La mortalidad aumenta con la edad, en China al 21 de marzo se reportó un $14.8 \%$ en $\geq 80$ años, y un $0 \%$ en $\leq 9$ años. Cifras menores a SARS-CoV con un $10 \%$ y MERS-CoV con $34 \%$, pero superior a la influenza estacional con un $0.01 \%-0.17 \%$ (Center for Evidence-Based Medicine; Ge et al.; Meng et al.; Sohrabi et al.).

Estudios han demostrado que, al igual que el SARS-CoV, se une al receptor de la enzima convertidora de angiotensina II (ECA II) de humanos, pero no puede unirse a células que no expresen dicho receptor. Los receptores ECA II son abundantes en el tracto respiratorio, sobre todo en las células alveolares de tipo II de pulmón, y en diversos órganos como corazón, riñón y/o gastrointestinal, entre otros. Dentro de la cavidad bucal los podemos encontrar altamente presentes en células epiteliales de la lengua $(40.6 \%$ de los presentes en la cavidad bucal) y menor medida en el conducto de las glándulas salivales y otros tejidos bucales y gingivales (Ge et al.; Peng et al.; Sabino-Silva et al.; 
Xu et al.). ECA II son objetivos tempranos de SARSCoV y probablemente también de COVID-19, debido a su alta similitud genómica, pero no se ha reportado en investigaciones. Los órganos con alta expresión de receptores ECA II debiesen ser consideradas como un riesgo potencial de infección (Peng et al.; SabinoSilva et al.; Xu et al.).

\section{MATERIAL Y MÉTODO}

Se realizó una revisión sistemática cualitativa. Los criterios de inclusión fueron estudios descriptivos y experimentales, in vivo y ex vivo, en humanos.

Los criterios de exclusión fueron estudios no relacionados con COVID-19, en animales y literatura gris. Se realizó una búsqueda sobre COVID-19, salud pública y odontología, en las bases de datos Medline y ClinicalKey. Los datos epidemiológicos actualizados fueron obtenidos de las páginas web de OMS y Centre for Evidence-Based Medicine (CEBM, 2020). Se utilizaron las palabras clave COVID-19, SARS-CoV-2, Coronavirus, Dentistry, Public Health.

\section{RESULTADOS}

1. Viabilidad de COVID-19 en el ambiente. Diversos estudios muestran las condiciones en las que pueden vivir diferentes tipos de coronavirus. HCoV, SARS-CoV, MERS-CoV, entre otros, pueden sobrevivir en el ambiente entre 2 horas a 9 días y persisten mejor en un ambiente con una humedad relativa del $50 \%$ que del 30\% (Ge et al.; Kampf et al.; Peng et al.; Van Doremalen et al.). Se comparó la viabilidad de los virus SARSCoV y SARS-CoV-2 en diversas superficies que se utilizan día a día. Se aplicaron los virus a una temperatura de $21^{\circ} \mathrm{C}$ a $23^{\circ} \mathrm{C}$ a un $40 \%$ de humedad relativa durante 7 días. Los resultados mostraron que el SARSCoV-2 es viable en aerosol durante 3 horas, en plástico durante 72 horas y en acero inoxidable por 48 horas, mismos resultados que SARS-CoV. La viabilidad en cobre fue 4 horas y 8 horas, y en cartón de 24 horas y 8 horas para SARS-CoV-2 y SARS-CoV respectivamente, aunque indican que la diferencia en el cartón debe ser interpretada con precaución. Las vidas medias del SARS-CoV-2 en aerosol es de $1.1 \mathrm{a}$ 1.2 horas, en acero inoxidable de 5.6 horas y en plástico de 6.8 horas (Van Doremalen et al.). Por esta razón se deben extremar las medidas precautorias en la consulta dental ya que las superficies contaminadas son frecuentemente contactadas siendo una vía de contagio potencial. Mantener limpio y seco el ambiente ayuda a disminuir la persistencia del COVID-19 y frecuentemente se deben limpiar y desinfectar el aire interior, piso, paredes y los accesorios como manijas de puertas, sillas y escritorios (Ge et al.; Kampf et al.; Meng et al.; Peng et al.).

2. Agentes biocidas efectivos. Diversos agentes biocidas pueden inactivar al SARS-CoV y MERS-CoV de forma efectiva, estos son el etanol (alcohol etílico) $78 \%$ - $95 \%$ durante 30 segundos, 2-propanol (alcohol isopropílico) $70 \%-100 \%$ durante 30 segundos, formaldehido $0.7 \%$ - $1 \%$ durante 2 minutos, povidona yodada $0,23 \%-7,5 \%$ durante 1 minuto. El glutaraldehído $2.5 \%$ durante 5 minutos o $0.5 \%$ durante 2 minutos inactiva al SARS-CoV, pero sin estudios para MERS-CoV. El hipoclorito de sodio requiere una concentración mínima de $0.21 \%$ durante 30 segundos para ser efectivo y el peróxido de hidrogeno una concentración de $0.5 \%$ durante un minuto, aunque estos resultados fueron con otros coronavirus y no MERS-CoV o SARS-CoV. La clorhexidina no es efectiva (Kampf et al.). La OMS recomienda utilizar hipoclorito de sodio $0.5 \%$ o alcohol etílico $70 \%$ durante, al menos, 1 minuto (World Health Organization \& Unicef, 2020).

3. El riesgo en la consulta dental. La consulta dental es una zona de riesgo extremo para el contagio de enfermedades como el COVID-19, prácticamente toda la práctica odontológica genera aerosoles. Hablar con el paciente, expulsa gotitas de saliva, el examen oral utilizando la jeringa triple que al expulsar aire y agua genera gotitas o bioaerosoles, contaminados con sangre, saliva u otros fluidos potencialmente infectados con microbios; al igual que el ultrasonido o instrumental rotatorio. En situaciones de pandemia debe evitarse todo instrumental y acción que genere aerosoles. Extreme las medidas de seguridad para el equipo dental y el paciente. Las gotitas grandes $(>5 \mu \mathrm{m})$ pueden contribuir a una transmisión a sujetos cercanos y otros sitios ingresando directamente a la conjuntiva ocular o la mucosa nasal y oral, la trasmisión a larga distancia es posible con bioaerosol o gotitas pequeñas $(\leq 5 \mu \mathrm{m})$, potencialmente infectadas, suspendidas en el aire siendo inhaladas o precipitando en el entorno generando infección cruzada entre pacientes y profesionales de salud, por esto, se deben tomar estrictos protocolos de prevención de infecciones para evitar infecciones nosocomiales por COVID-19 logrando bloquear la 
GUIÑEZ, C. M. Impacto del COVID-19 (SARS-CoV-2) a nivel mundial, implicancias y medidas preventivas en la práctica dental y sus consecuencias psicológicas en los pacientes. Int. J. Odontostomat., 14(3):271-278, 2020.

trasmisión de persona a persona, especialmente en pacientes con periodo de incubación/asintomáticos que lo desconocen o prefieren ocultarlo, ver Fig. 2 (Ge et al.; Guo et al.; Li et al.; Meng et al.; Peng et al.; Qu \& Zhou; Sabino-Silva et al.; Tang et al.).

Es frecuente utilizar radiografías intraorales las que pueden aumentar el flujo salival y/o provocar tos o nauseas; siempre que sea posible se deben reemplazar por radiografías extraorales como panorámica o CBCT mientras dure el brote de COVID-19 (Guo et al.; Li et al.; Meng et al.; Qu \& Zhou; Sabino-Silva et al.; Tang et al.).

Desde que comenzó el brote, en China, solamente atienden emergencias con una estricta implementación de control y prevención de infecciones (Ge et al.; Guo et al.; Li et al.; Meng et al.; Peng et al.; Qu \& Zhou; Tang et al.).

4.La influencia psicológica de una pandemia en la atención dental. En odontología existen diversas enfermedades con un componente psicosomático las cuales pueden empeorar en una situación de pandemia, tales como enfermedades de la mucosa oral, periodontal, bruxismo, entre otras, en las cuales se debe prestar atención a la relación entre las enfermedades orales y el estado psicológico de los pa- cientes para evaluar las medidas correspondientes. Las emergencias de salud pública pueden generar estrés psicológico como ansiedad, tensión, irritabilidad, depresión, ilusiones patológicas, alucinaciones, delirios, anorexia, náuseas, diarrea, poliuria, sudoración, tensión muscular, entre otros. Estos síntomas pueden aparecer desde el día siguiente a la emergencia y el impacto psicológico puede durar hasta varios años. Es importante incorporar la intervención psicológica durante la crisis para reducir el riesgo de estos trastornos (Qu \& Zhou).

Los trastornos psicosomáticos orales son aquellos sin causa determinada y pueden provocar dolor oral crónico, molestias y sequedad bucal. Incluyen síndrome de boca urente, odontalgia atípica, halitofobia, fobia dental, entre otros. Estas patologías se consideran causadas por factores emocionales y es importante aliviar el dolor físico y mental, los que pueden empeorar con el estrés psicológico. La ulcera recurrente oral o liquen plano son patologías idiopáticas y se considera que están estrechamente relacionados con factores psicológicos. Los eventos de estrés pueden causar un aumento de 3 veces los ataques por ulceras recurrentes orales y pueden aumentar su gravedad. El síndrome de boca urente presenta ardor sin causa clínica clara y puede dificultar la dieta y el habla generando un importante impacto

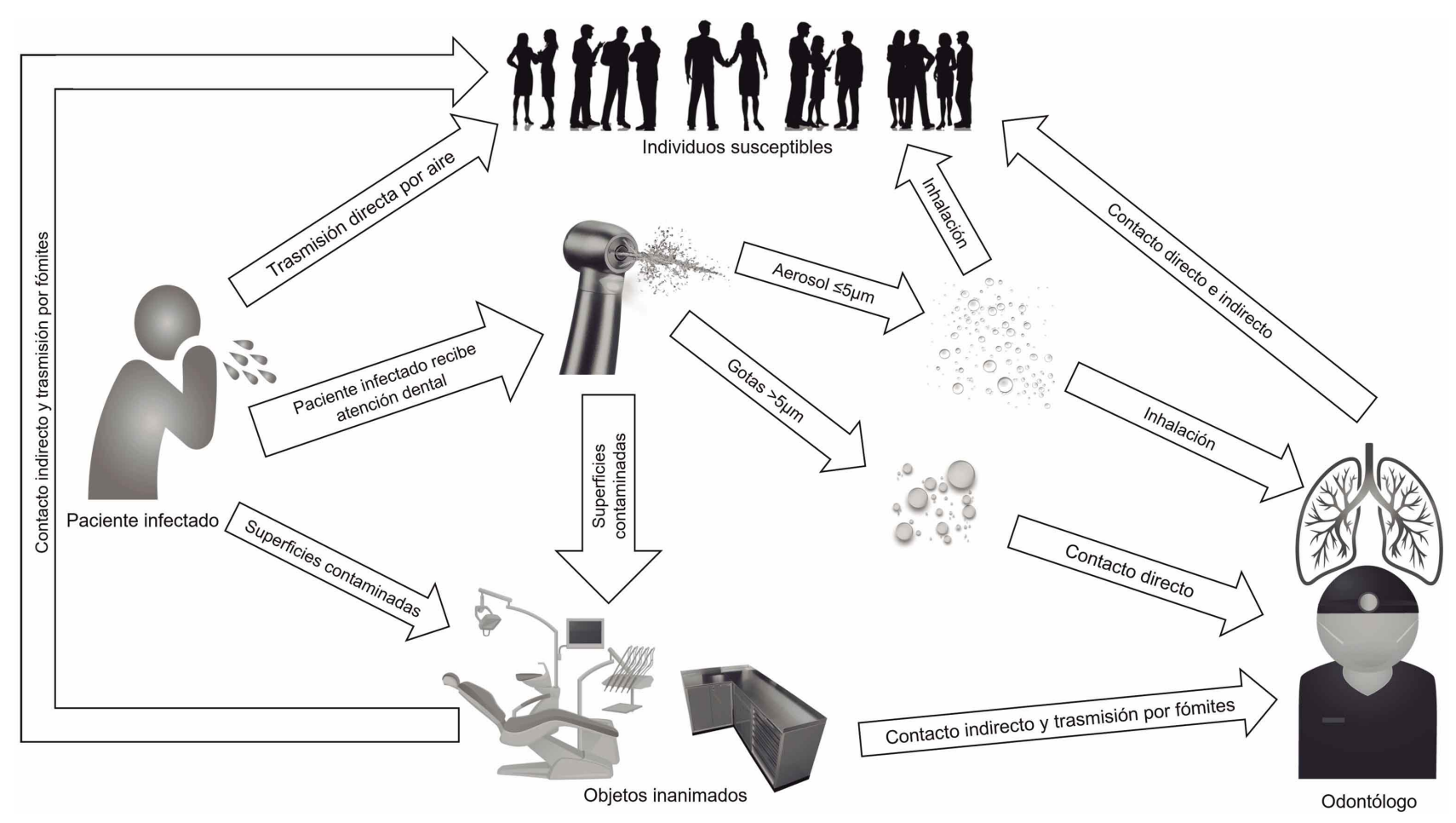

Fig. 2. Posibles vías de contagio de COVID-19 en la consulta dental. 
GUIÑEZ, C. M. Impacto del COVID-19 (SARS-CoV-2) a nivel mundial, implicancias y medidas preventivas en la práctica dental y sus consecuencias psicológicas en los pacientes. Int. J. Odontostomat., 14(3):271-278, 2020

negativo en la calidad de vida, los factores psicológicos son su principal causa. Estudios ha mostrado un aumento de la xerostomía en un $51 \%$ en pacientes con ansiedad, $47 \%$ con depresión, $41 \%$ con trastorno bipolar y $39 \%$ con esquizofrenia, siendo significativamente mayor en pacientes con algún trastorno mental respecto a pacientes sanos (Qu \& Zhou).

El bruxismo está relacionado con las emociones, entre otras causas, se ha visto que mientras mayor sea la ansiedad es mayor el bruxismo. Por otro lado, se ha observado una correlación positiva entre el estrés/factores psicológicos y la enfermedad periodontal, los altos niveles de cortisol séricos están significativamente relacionados con la periodontitis crónica. Las enfermedades mentales también afectan al riesgo de caries y cantidad de dientes perdidos, siendo una relación bidireccional, un metaanálisis demostró que todos los diagnósticos psiquiátricos se relacionaron positivamente con mayor número de caries y restauraciones (Qu \& Zhou).

5. Prevención de COVID-19. La literatura no reporta recomendaciones clínicas específicas para el COVID-19 en odontología, pero se han creado diversos protocolos para prevenir la infección y la propagación de esta pandemia. El centro de salud de enfermedades de corea del sur (KCDC) entrega 6 consejos para la población general las que incluyen el lavado de manos frecuente; si presenta tos, estornudo o cualquier síntoma respiratorio, y no tiene mascarillas, debe cubrirse la boca y nariz con el codo o con una toalla de papel la cual debe desechar y lavarse las manos; no toque sus ojos, nariz y/o boca con sus manos; utilice mascarilla cuando visite las instalaciones de salud; no visite lugares concurridos; no toque personas que presenten síntomas como fiebre o tos. Estas medidas pueden ser seguidas por toda la población, excepto la última que los equipos de salud no pueden cumplir al atender los casos sospechosos/comprobados (Ge et al.; Huh et al.).

5.1.Lavado de manos. El lavado de manos es fundamental para la prevención de cualquier enfermedad trasmisible, debe ser frecuente con jabón y agua durante 30 segundos o más, si no están disponibles, utilice alcohol gel $70 \%$ - $90 \%$ ya que ambas formas son efectivas, pero si existe suciedad visible en las manos debe lavarlas con agua y jabón. En la práctica dental un lavado de manos rutinario es un prerrequisito el cual debe efectuarse " 2 antes y 3 después" que consiste en antes de examinar al paciente y antes de un procedimiento dental/aséptico.
Después de tocar las superficies y equipo sin desinfección; después de tocar al paciente; y después de tocar la mucosa oral, piel dañada, sangre, fluidos corporales, secreciones y excretas (Ge et al.; Huh et al.; Kampf et al.; Li et al.; Meng et al.; Peng et al.; SabinoSilva et al.; Sohrabi et al.; Tang et al.).

5.2. Valoración de pacientes. Toda persona que ingrese al centro de salud debe usar mascarillas. Se debe fortalecer las áreas públicas, preselección de pacientes y TRIAGE con personal equipado con EPP y desinfectantes para uso personal e instrumental. Deben realizar un preexamen antes de ingresar al box dental en donde se toma la temperatura del paciente y todo acompañante, incluso los que estén en el automóvil, siendo fuertemente recomendado el uso de termómetro sin contacto. Luego se realiza una investigación y registro de antecedentes epidemiológicos con un cuestionario que puede incluir las siguientes preguntas: (1) ¿ha experimentado fiebre en los últimos 14 días? ¿usa antipiréticos?, (2) ¿ha experimentado algún problema respiratorio reciente, como tos o dificultad respiratoria en los últimos 14 días?, (3) ¿ha viajado o visitado lugares con casos de COVID-19? (4) ¿Ha estado en contacto con pacientes confirmados con COVID-19 en los últimos 14 días?, (5) ¿Ha estado en contacto con personas que hayan viajado a lugares con casos de COVID19 , o en zonas con casos recientes de fiebre o problemas respiratorios en los últimos 14 días?, (6) ¿Ha estado en contacto con dos personas con fiebre o problemas respiratorios en los últimos 14 días?, (7) ¿Ha participado recientemente en cualquier reunión o estado en contacto cercano con muchas personas desconocidas? (Ge et al.; Huh et al.; Li et al.; Meng et al.; Peng et al.; Tang et al.).

Si el paciente responde "sí" en cualquiera de las preguntas con temperatura $\angle 37,3^{\circ} \mathrm{C}$ se puede referir el tratamiento y recomendar una auto cuarentena. Si responde "sí" a cualquiera de las preguntas con temperatura $\geq 37,3^{\circ} \mathrm{C}$ debe ser inmediatamente enviado a cuarentena y reportado al centro de control de enfermedades correspondiente. Si el paciente responde "no" a todas las preguntas con temperatura $<37,3^{\circ} \mathrm{C}$ se puede tratar con todas las medidas de protección extra evitando procedimientos que generen salpicaduras o aerosoles, pero si no es emergencia se recomienda diferir. Si el paciente responde "no" a todas las preguntas con temperatura $\geq 37,3^{\circ} \mathrm{C}$ debe evaluarse la causa de la fiebre, si no se reconoce debe ser derivado a un centro de salud para evaluar posibilidad de COVID-19. Los que aprueban de- 
ben pasar ordenadamente al área de espera, correctamente ventilada, sin acompañante, excepto los niños que puede acompañarlos un adulto, con una distancia mínima de 1 metro entre personas. Los acompañantes deben quedarse fuera del área de espera o del hospital. (Ge et al.; Li et al.; Meng et al.; Peng et al.; Tang et al.).

5.3. Protocolos para el odontólogo. Se deben proteger todas las vías de trasmisión utilizando el EPP, realizar el lavado de manos antes de colocarse el EPP (los guantes deben ser el último elemento en colocarse) y " 2 antes y 3 después". No debe comer ni beber en el box dental ni colocar elementos personales en el box, no usar guantes para tocar directamente el entorno. Al retirar el EPP primero quítese los guantes, lávese las manos para continuar con el EPP restante (Ge et al.; Huh et al.; Li et al.; Meng et al.; Peng et al.; Sabino-Silva et al.; Sohrabi et al.; Tang et al.).

5.4. Elementos de protección personal. Las mascarillas quirúrgicas pueden proporcionar protección en la práctica dental diaria, pero no contra gotitas $<5 \mu \mathrm{m}$ o Mycobacterium tuberculosis, por ejemplo. Según la OMS, se deben usar mascarillas N95 o FFP2 al tratar a pacientes con COVID-19 las que deben cubrir completamente la nariz, barbilla y ajustarse bien a la cara, no usar otra mascarilla en el interior y su uso continuo no debe exceder las 4 horas ( $\mathrm{Li}$ et al.). Ver la Tabla I para recomendaciones de EPP.

Todo el personal, incluido limpieza y seguridad, deben utilizar la protección correspondiente a su puesto de trabajo. Prepare anticipadamente el EPP a utilizar y hágalo de forma adecuada, el cabello debe estar atado, no debe quedar cabello suelto y las joyas, relojes o accesorios deben removerse para evitar su contaminación; beba agua para evitar la deshi- dratación posterior y vaya al baño antes de usar el EPP; si encuentra contaminación o daños después del uso debe reemplazarlo; debe cambiar los guantes si están mojados. No toque el EPP y áreas sin protección con las manos durante el tratamiento y evitar tocar la superficie contaminada del EPP al retirarlo. Las batas de aislación se deben sacar al salir del box dental. Las antiparras y mascaras faciales deben limpiarse y desinfectarse con alcohol $75 \%$ o sumergirse en $500 \mathrm{mg}-1000 \mathrm{mg} / \mathrm{L}$ de cloro durante 30 minutos, enjuagarse y secarse para utilizarse como reemplazo. Cambie la ropa de trabajo al terminar la jornada y se recomienda ducharse al regresar a casa (Huh et al.; Li et al.; Tang et al.). El eczema en la cara es una complicación frecuente frente al uso prolongado del EPP; para aliviar los síntomas, puede utilizar cremas o ungüentos medicados. Parchar la piel con vendaje adhesivo es uno de los métodos preventivos (Huh et al.).

5.5. Medidas en la atención dental.El trabajo a cuatro manos ayuda a mejorar la calidad, eficiencia y controlar las infecciones, si no es posible los asistentes deben preparar equipos y artículos relacionados con las necesidades del tratamiento, utilizar succión de alto volumen (hemosuctor) y succión débil para aspirar saliva y evitar generar aerosoles o gotas, el paciente no debe morder la succión y el mango de succión debe ubicarse lo más lejos posible debajo de la boca del paciente. El examen oral debe ser relajado evitando causar tos o reflejos faríngeos, use el eyector para eliminar la saliva a tiempo (Li et al.).

5.6. Uso de colutorios. Los colutorios previos a la atención dental ayudan a disminuir la cantidad de aerosol, un metaanálisis concluyó que su uso provocó una reducción promedio de un $68.4 \%$ de unidades formadoras de colonias en el aerosol dental (Ge et al.). El peróxido de hidrogeno $1 \%$, povidona $0,2 \%$ o

Tabla I. Recomendaciones para atención de pacientes según guías de atención en China y Corea del sur (Huh et al.; Li et al.; Meng et al.; Peng et al.; Tang et al.)

\begin{tabular}{|c|c|c|c|c|c|c|c|c|c|}
\hline Situación & $\begin{array}{l}\text { Mascarilla } \\
\text { Quirúrgica }\end{array}$ & $\begin{array}{l}\text { Mascarilla } \\
\text { N95/FFP2 o } \\
\text { equivalente }\end{array}$ & $\begin{array}{l}\text { Mascara } \\
\text { facial }\end{array}$ & Antiparras & $\begin{array}{l}\text { Guantes } \\
\text { Desechables de } \\
\text { Látex* }^{*}\end{array}$ & $\begin{array}{l}\text { Bata de aislación } \\
\text { desechable }\end{array}$ & $\begin{array}{l}\text { Ropa } \\
\text { protectora }\end{array}$ & $\begin{array}{l}\text { Gorro } \\
\text { desechable }\end{array}$ & $\begin{array}{l}\text { Cubre } \\
\text { calzado }\end{array}$ \\
\hline TRIAGE & $\sqrt{ }$ & s & s & s & $\checkmark$ & $\mathrm{N}$ & N & $\sqrt{ }$ & $\mathrm{N}$ \\
\hline Examen oral & $\sqrt{ }$ & s & s & $\sqrt{ }$ & $\sqrt{ }$ & $\mathrm{N}$ & N & $\sqrt{ }$ & s \\
\hline Generación de aerosoles & $\mathrm{N}$ & $\sqrt{ }$ & $\sqrt{ }$ & $\sqrt{ }$ & $\sqrt{ }$ & $\checkmark$ & $\sqrt{ }$ & $\sqrt{ }$ & $\sqrt{ }$ \\
\hline $\begin{array}{l}\text { Admisión de pacientes sospechosos o } \\
\text { confirmados }\end{array}$ & $\mathrm{N}$ & $\sqrt{ }$ & $\checkmark$ & $\checkmark$ & $\checkmark$ & $\checkmark$ & N & $\sqrt{ }$ & $\sqrt{ }$ \\
\hline Limpieza y desinfección de la habitación & $\mathrm{N}$ & $\sqrt{ }$ & $\checkmark$ & $\checkmark$ & $\sqrt{ }$ & $\checkmark$ & $\sqrt{ }$ & $\sqrt{ }$ & $\sqrt{ }$ \\
\hline
\end{tabular}

$\sqrt{ }$ : EPP recomendado; S: EPP de uso selectivo; N: EPP no recomendado. *Se deben usar guantes dobles teniendo en cuenta el riesgo de rotura de los guantes o el riesgo de exposición a infecciones al realizar tratamientos médicos, cuidados de enfermería, pruebas y limpieza de áreas de pacientes sospechosos y confirmados 
cloruro de cetilpiridinio (CPC) $0.05 \%-0.1 \%$, al contener agentes oxidativos, son efectivos contra diversos tipos de coronavirus, pero debemos recordar que no hay datos específicos para SARS-CoV-2. Su uso se recomienda si no es posible utilizar goma dique (Li et al.; Meng et al.; Peng et al.).

5.7. Uso de goma dique. La goma dique disminuye un $70 \%$ la producción de aerosol o salpicaduras contaminadas con saliva o sangre y en $90 \%$ los microorganismos en el aire en un diámetro de 1 metro. Debe acompañarse con succión de alto volumen para el aerosol y succión regular para salpicaduras, necesitando el trabajo a cuatro manos. Si no es posible aislar de forma absoluta, como en procedimientos subgingivales, se recomienda utilizar instrumentos manuales o químicos y no instrumental rotatorio o ultrasonido para minimizar la generación de aerosol (Ge et al.; Meng et al.; Peng et al.).

5.8. Manejo del box dental. El área de diagnóstico y tratamiento debe tener buena ventilación abriendo la ventana al menos 3 veces al día durante 30 minutos, desinfectar el área e instrumental regularmente, si es necesario utilice radiación UV 2 veces al día durante 30 minutos, aumente el intervalo entre los pacientes y elimine todos los desechos médicos después de la atención. En Box dentales con más de un sillón contiguos organice un solo sillón para las atenciones y coloque la menor cantidad de artículos en los mesones. Después de la atención desinfecte con un trapo húmedo con $2100 \mathrm{mg} / \mathrm{L}$ de cloro y en casos sospechosos utilice $5000 \mathrm{mg} / \mathrm{L}$ las superficie y el piso (Ge et al.; Li et al.; Tang et al.; World Health Organization).

5.9. Tipos de atención dental. En áreas en donde se está extendiendo la pandemia es difícil diferenciar pacientes asintomáticos de sanos por lo que se recomienda solo atender emergencias como dolor dental agudo, trauma dental, trauma oral y maxilofacial e infecciones maxilofaciales graves, utilizando el EPP necesario, goma dique y succión de saliva de alto volumen para disminuir aerosoles o salpicaduras, prefiriendo utilizar instrumental manual, remoción de caries químico-mecánica y restauraciones provisorias o de vidrio ionómero. En caso de exodoncias prefiera suturas reabsorbibles para disminuir las sesiones. Irrigue con agujas suavemente y aspirando para disminuir la pulverización. De forma alternativa, los pacientes pueden ser atendidos en una sala aislada y con buena ventilación; las habitaciones con presión negativa deben estar disponibles para los pacientes sospechosos de COVID-19. En casos que amenacen la vida, el paciente debe ser admitido en un hospital inmediatamente y realizar TC de tórax, si está disponible, porque la prueba RT-PCR tarda mucho tiempo. Los pacientes con traumatismos o infecciones maxilofaciales generalmente presentan una temperatura elevada lo que debe diferenciarse de COVID19 con la historia epidemiológica, etiología, examen clínico, examen de sangre y TC de tórax (Li et al.; Meng et al.).

\section{DISCUSIÓN}

Estudios demuestran que la posibilidad de infección por aerosol y fómites es real, desde que se esparcen los virus su viabilidad, por ende, la posibilidad de contagio por aerosol es de horas y en las superficies de días (Van Doremalen et al.), por esta razón debemos extremar las precauciones en la práctica odontológica evitando generar aerosoles, desinfectando las superficies, ambientes e instrumental reutilizable correctamente, y siendo cuidadosos con la correcta implementación de los EPP.

Debido a que la mayoría de las enfermedades orales no son autolimitadas, se debe tomar consciencia que suspender la atención electiva puede afectar potencialmente la psicología del paciente, especialmente enfermedades psicosomáticas. El realizar consultas en línea y orientación gráfica puede mejorar la respuesta de estos pacientes aliviando el estrés psicológico que también provoca el aislamiento en el hogar (Qu \& Zhou).

Todavía falta información sobre el COVID-19, no existen estudios que demuestren que la fase de recuperación no presenta un riesgo potencial de trasmisión recomendándose postergar el diagnóstico y tratamiento odontológico electivo en un mes posterior a superar la enfermedad por COVID-19 (Li et al.). No hay estudios de agentes biocidas específicos para COVID-19 ni protocolos probados enfocados en tratar esta nueva pandemia y solamente se están adaptado protocolos y mecanismos de otros brotes virales similares, como el SARS-CoV.

Agradecimientos. Agradezco a mis padres por la inspiración y motivación a realizar este estudio debido al impacto severo que genera en la salud pública a nivel mundial siendo una batalla que debemos luchar y ganar como humanidad. 
GUIÑEZ, C. M. Impact of COVID-19 worldwide, implications and preventive measures in dental practice and its psychological consequences on patients. Int. J. Odontostomat., 14(3):271-278, 2020.

ABSTRACT: In December 2019, a new outbreak of pneumonia emerged in China, which was identified as SARSCoV-2, which has a rapid spread, being transmitted mainly through cough, sneeze or contact. It has various symptoms such as fever, dry cough and myalgia. It can affect at any age, being mainly mild and with spontaneous resolution, but in older people and/or with comorbidities, it can present a severe or fatal course. For its diagnosis it's important to evaluate the presence of fever, carry out an epidemiological history, chest CT and laboratory tests such as RT-PCR or samples from the respiratory tract. A qualitative systematic review was performed by searching the Medline and ClinicalKey databases, 72 articles were found, which were read in full, of which 16 met the inclusion and exclusion criteria.Most of the dental actions produce drops or aerosol, for this reason protective measures must be taken, patient selection and types of treatments to decrease the possibility of infections in health personnel and patients, evaluate the use of rubber dam and preoperatorial mouthrinses avoid aerosol-generating instruments, postpone elective care, treat only dental emergencies and take extreme disinfection measures at facilities using effective biocidal agents against coronavirus. The transmission by fomites and aerosols is proven so we must take extreme precautions. In pandemic situations, consideration should be given to providing psychological support to patients, especially with psychosomatic oral pathologies, which may worsen. The possibility of transmission in the recovery period is neither proven nor ruled out.

KEY WORDS: COVID-19, SARS-CoV-2, Coronavirus, Dentistry, Public Health.

\section{REFERENCIAS BIBLIOGRÁFICAS}

Baud, D.; Qi, X.; Nielsen-Saines, K.; Musso, D. Pomar, L. \& Favre, G. Real estimates of mortality following COVID-19 infection. Lancet Infect. Dis., 2020. DOI: https://doi.org/10.1016/S14733099(20)30195-X

Centre for Evidence-Based Medicine (CEBM). Global Covid-19 Case Fatality Rates. Oxford, Centre for Evidence-Based Medicine, 2020. Disponible en: https://www.cebm.net/global-covid-19-case-fatalityrates/

Ge, Z. Y.; Yang, L. M.; Xia, J. J.; Fu, X. H., Zhang, Y. Z. Possible aerosol transmission of COVID-19 and special precautions in dentistry. $J$. Zhejiang Univ. Sci. B, 2020. DOI: https://doi.org/10.1631/ jzus.B2010010

Guo, H.; Zhou, Y.; Liu, >X. \& Tan, J. The impact of the COVID-19 epidemic on the utilization of emergency dental services. J. Dent. Sci., 2020. DOI: https://doi.org/10.1016/j.jds.2020.02.002

Huh, S. How to train health personnel to protect themselves from SARSCoV-2 (novel coronavirus) infection when caring for a patient or suspected case. J. Educ. Eval. Health Prof., 17:10, 2020. DOI: https:/ /doi.org/10.3352/jeehp.2020.17.10

Kampf, G.; Todt, D.; Pfaender, S. \& Steinmann, E. Persistence of coronaviruses on inanimate surfaces and their inactivation with biocidal agents. J. Hosp. Infect., 104(3):246-51, 2020.

Meng, L.; Hua, F. \& Bian, Z. Coronavirus disease 2019 (COVID-19): emerging and future challenges for dental and oral medicine. J. Dent. Res., 2020. Available from: https://www.unboundmedicine.com/ medline/citation/32162995/Coronavirus_Disease_2019_(COVID19): Emerging and Future Challenges for Dental and Oral Medicine

Pan, X.; Ojcius, D. M.; Gao, T.; Li, Z.; Pan, C. \& Pan, C. Lessons learned from the 2019-nCoV epidemic on prevention of future infectious diseases. Microbes. Infect., 22(2):86-91, 2020.

Peng, X.; Xu, X.; Li, Y.; Cheng, L.; Zhou, X. \& Ren, B. Transmission routes of 2019-nCoV and controls in dental practice. Int. J. Oral Sci., 12:9, 2020. DOI: https://doi.org/10.1038/s41368-020-0075-9

Qu, X. \& Zhou, X. D. Psychological intervention in oral patients in novel coronavirus pneumonia outbreak period. Zhonghua Kou Qiang $Y_{i}$ Xue Za Zhi, 55(0):E003, 2020. DOI: https://doi.org/10.3760/ cma.j.cn112144-20200213-00053

Sabino-Silva, R.; Jardim, A. C. G. \& Siqueira, W. L. Coronavirus COVID19 impacts to dentistry and potential salivary diagnosis. Clin. Oral Invest/G., 2020. DOI: https://doi.org/10.1007/s00784-020-03248-x

Sohrabi, C.; Alsafi, Z.; O'Neill, N.; Khan, M.; Kerwan, A.; Al-Jabir, A.; Iosifidis, C. \& Agha, R. World Health Organization declares global emergency: A review of the 2019 novel coronavirus (COVID-19). Int. J. Surg., 76:71-6, 2020.

Tang, H. S.; Yao, Z. Q. \& Wang, W. M. Emergency management of prevention and control of novel coronavirus pneumonia in departments of stomatology. Zhonghua Kou Qiang Yi Xue Za Zhi, 55(0):E002, 2020. DOI: https://doi.org/10.3760/cma.j.cn11214420200205-00037

van Doremalen, N.; Bushmaker, T.; Morris, D. H.; Holbrook, M. G.; Gamble, A.; Williamson, B.; Tamin, A.; Harcourt, J.; Thornburg, N.; Gerber, S.; Lloyd-Smith, J.; de Wit, E. \& Munster, V. J. Aerosol and surface stability of SARS-CoV-2 as compared with SARS-CoV-1. N. Engl. J. Med., 2020. DOI: https://doi.org/10.1056/NEJMc2004973

World Health Organization (WHO). Coronavirus disease 2019 (COVID19) Situation Report - 65. Ginebra, World Health Organization, 2020. Disponible en: https://www.who.int/docs/default-source/coronaviruse/ situation-reports/20200325-sitrep-65-covid19.pdf?sfvrsn=ce13061b 2

World Health Organization \& UNICEF. Water, sanitation, hygiene and waste management for the COVID-19 virus. Technical brief, 3 March 2020. Ginebra, World Health Organization, 2020. Disponible en: https://apps.who.int/iris/bitstream/handle/10665/331305/WHO-2019NcOV-IPC_WASH-2020.1-eng.pdf

Xu, H.; Zhong, L.; Deng, J.; Peng, J.; Dan, H.; Zeng, X.; Li, T. \& Chen, Q. High expression of ACE2 receptor of 2019-nCoV on the epithelial cells of oral mucosa. Int. J. Oral Sci., 12:8, 2020. DOI: https://doi.org/ $10.1038 / s 41368-020-0074-x$

Dirección para correspondencia:

Dr. Marcial Guiñez Coelho

Cirujano Dentista, Clínica Gama Smile

Santiago - CHILE

Email: marcial.guinezc@gmail.com

Recibido : 26-03-2020

Aceptado: 27-03-2020 